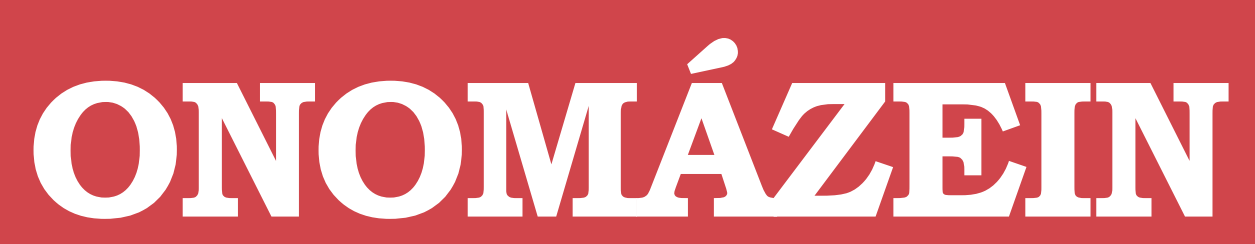

Revista de lingüística, filología y traducción
PONTIFICIA UNIVERSIDAD

\title{
La función apelativa del marcador discursivo venga en Twitter
}

Appellative function of the discourse markervenga in Twitter

\section{Salvador López Quero}

Universidad de Córdoba

España

\section{(C) $($ (i) $\ominus$}

Salvador López Quero: Departamento de Ciencias del Lenguaje, Universidad de Córdoba, España. | Correo electrónico: salvador.lopez.quero@uco.es 


\section{Resumen}

El marcador conversacional venga se usa en Twitter en intervenciones no reactivas para transmitir ánimo o introducir una petición, y en intervenciones reactivas para expresar desacuerdo, valores derivados de su uso imperativo originario. Mayoritariamente, venga ocupa la posición inicial del enunciado y se usa, sobre todo, para transmitir ánimo en enunciados cargados de cortesía positiva. Este marcador, por tanto, potencia las relaciones sociales. La fuerza pragmática del acto ilocutivo depende del valor más o menos efectivo de los intensificadores que en cada situación comunicativa acompañen al marcador: el vocativo implicativo y determinadas expresiones imperativas son los principales intensificadores de la función apelativa. Sin embargo, en los enunciados irónicos se aminora la función apelativa. Otros marcadores discursivos en coocurrencia con venga — vamos, va, anda y pues - intensifican sobre todo el ánimo (Venga, vamos / Vamos, venga), la petición (Venga va / Pues venga) o el desacuerdo (Venga, anda / Anda, venga).

Palabras clave: Twitter; venga; ánimo; petición; desacuerdo.

\section{Abstract}

Discourse marker venga is used in Twitter in non-reactive interventions in order to cheer up or present a request, and in reactive interventions to express disagreement, values derived from its initial imperative use. Venga mainly holds the first position in the statement and is used, especially, to cheer up in statements with positive politeness. Thus, this marker strengthens social relationships. The pragmatic force of the illocutionary act depends on the more or less effective value of the intensifiers that go with the marker in each communicative situation: implicative vocative and certain imperative expressions are the main intensifiers of the appellative function. Nevertheless, appellative function is reduced in ironic statements. Other co-occurrence discourse markers with venga_vamos, va, anda and pues-intensify mostly mood (Venga, vamos / Vamos, venga), request (Venga va / Pues venga) or disagreement (Venga, anda / Anda, venga).

Keywords: Twitter; venga; mood; request; disagreement. 


\section{Introducción}

En este trabajo, dedicado al estudio de la función apelativa del marcador discursivo venga en la red social Twitter, se pondrán al descubierto — dentro de la polifuncionalidad discursiva que caracteriza a este marcador conversacional-cuáles de sus funciones pragmáticas guardan relación con la función apelativa del lenguaje tan característica de venga en Twitter.

Para Baran (2014: 8), venga, vaya, anda y toma son "marcadores conversacionales interactivos", conocidos en la tradición gramatical como "imperativos gramaticalizados". Y, más recientemente, Ciarra (2016: 137) clasifica venga como "marcador discursivo conversacional de interacción”. En cuanto a su función pragmática, Matte Bon (1992: 320) ya había afirmado que venga se usa "para incitar a alguien a que haga algo o para darle ánimos". Y Casado Velarde (1995), que lleva a cabo una clasificación de los marcadores textuales de acuerdo con los diferentes tipos de sentido, asocia venga con llamada de atención. Se trata, de acuerdo con Calsamiglia y Tusón (1999: 249), así como con Cortés Rodríguez (2001: 544), de la función para estimular al interlocutor. Y en el Diccionario de partículas del español se afirma que "llama la atención del destinatario y le anima a actuar según lo que se va a decir o solicitar a continuación o lo que se ha dicho o solicitado anteriormente" (Briz y otros, 2008). Por último, Baran (2010) distingue (entre otras) la función yusiva o exhortativa, la función de partícula reactiva de protesta, muestra de desagrado o rechazo, etc.

En cuanto al origen de este marcador conversacional, Daniels (2014) afirma que de su uso imperativo derivan los valores apelativo y de desacuerdo. Téngase en cuenta que, aunque el desacuerdo parte de la subjetividad y desempeña una función expresiva, el emisor intenta influir en el comportamiento del destinatario, por lo que también se lleva a cabo la función apelativa. Y, con respecto a la gramaticalización, Luque Toro (2009: 140) insiste en "la idea de mandato sobre la que se desarrolla su gramaticalización y en que se trata de una forma impulsiva característica de la oralidad". No obstante, como Blas Arroyo afirma, "el empleo como verbo está completamente diluido" (2001 [1998]: 7). El proceso de gramaticalización que experimentan los verbos de movimiento españoles (vamos, venga, vaya, anda) - advierte Baran (2014: 9) - puede describirse igualmente "desde la perspectiva de la subjetivización dado que [sic] el cambio lingüístico ante el que nos hallamos conduce a la puesta en relieve de las actitudes y de las valoraciones de los hablantes ante lo comunicado o ante la situación comunicativa en la que se ven implicados los interactantes".

En este trabajo se demostrará que el uso de venga en Twitter se da, sobre todo, en intervenciones no reactivas que expresan petición o ánimo, y en intervenciones reactivas que expresan desacuerdo. Se trata del valor más próximo al valor conativo de la forma imperativa originaria. Téngase en cuenta, no obstante, que no es fácil en todos los enunciados delimitar la petición del ánimo, ya que a veces se dan conjuntamente estos dos actos de habla. 
Esta investigación se basa en un corpus de 500 tuits, recogidos desde mayo de 2015 hasta agosto de 2016. En este corpus el marcador ocupa mayoritariamente la posición inicial (51.94\%); en segundo lugar, la posición final (28.62\%), y con 19.43\%, la medial. Metodológicamente, es el orden que se va a seguir en este trabajo.

\section{El marcador discursivo conversacional venga en posición inicial del enunciado}

\subsection{Venga para introducir una petición}

El miembro del discurso al que afecta venga suele ser una oración con el núcleo verbal con valor imperativo. Para Haverkate (2003: 65-66), la frase en imperativo en español, en condiciones estándar, se interpreta como petición y no como orden. También, afecta con frecuencia a un vocativo y, a veces, tanto al imperativo como al vocativo, elementos gramaticales que intensifican la función apelativa del lenguaje. Téngase en cuenta que nuestro venga se corresponde con lo que Alonso-Cortés denominó interjecciones instativas, "que acompañan a un imperativo indicando la fuerza ilocutiva del acto instativo” (1999: 4028). La fuerza pragmática del acto ilocutivo que introduce el marcador viene determinada por la estructura lingüística del enunciado: cuando a través de venga se infiere la función apelativa no suele ser el marcador mismo el que formula la petición, el ánimo o el desacuerdo, sino que a través de venga se infiere el acto de habla ilocutivo de petición, ánimo o desacuerdo. En este sentido, de acuerdo con la propuesta formulada por Baran, venga es una de las marcas de carácter interactivo que, "aunque no inciden en el contenido informativo de los enunciados, determinan en gran medida la naturaleza de los actos de habla sobre los que se realizan y se presentan" (Baran, 2014: 7). Según Monjour, al referirse a los por él llamados introductores exhortativos, "sirven muchas veces para acompañar e introducir la petición, aunque no sea fácil determinar si contribuyen a atenuar o a intensificar el enunciado" (2006: 35). En esta argumentación discursiva cabe hablar de diferentes grados de intensificación. Por ejemplo, en los enunciados siguientes la fuerza ilocutiva es máxima y el valor de la petición tiene carácter exhortativo:

(1) Venga, vamos a conseguirlo!! \#MadridALalzquierda

(2) iVenga no seas tímido! Envíanos la conversación entre \#EIPirata y Brian y consigue \#RockFMEntradasACDC

(3) VENGA CONOCER NUESTRA REVISTA 100\% DEDICADA A DANNA PAOLA! https://dreamersdedanna.wordpress.com/revista/

(4) venga disparar selfies detrás de las cámaras \#masterchef

(5) Venga, reventad a likes este tweet y os la enseño :p A ver vuestro interés!! >:D 
Téngase en cuenta, como norma general, que no siempre es fácil determinar si en el enunciado predomina la petición o el ánimo. Sirva (1) como ejemplo, en el que se amalgaman la petición y el ánimo. Por otra parte, nótese cómo en los enunciados anteriores el acto de habla directivo exhortativo se consigue por la fuerza pragmática de las formas verbales: perífrasis ingresiva de contenido proposicional exhortativo, subjuntivo negativo exhortativo y tres imperativos, con error gramatical en (3) y (4), y por las estructuras oracionales exclamativas. Todo este conjunto de recursos lingüísticos actúa a modo de intensificadores de la función apelativa que se infiere a través del marcador venga. Por lo tanto, la fuerza pragmática depende del valor más o menos efectivo de los intensificadores que en cada momento acompañen al marcador. En cuanto a la argumentación discursiva, en todos los enunciados anteriores al marcador sigue inmediatamente la conclusión (Venga + conclusión). Y, con respecto a la objetividad/subjetividad derivada del empleo del modo indicativo o subjuntivo, Luque Toro advierte que la esencia de mandato se presenta con la doble modalidad indicativo/subjuntivo: con el primero se señala un aviso o advertencia, mientras que con el subjuntivo "la idea dominante es la de hacer prevalecer la voluntad del hablante ("querer que") para que se lleve a cabo la acción que está en curso, también a veces con un cierto matiz negativo centrado en la relación hablante/oyente según el contexto, es decir, 'no quiero que'” (2009: 140-141). Por ejemplo, en (2) cabe la interpretación no quiero que seas tímido, así como en (7), (8) y (11) de los enunciados que siguen las interpretaciones son quiero que deje ese orgullo, no quiero que le ruegue más y quiero que usemos el HT.

Por otra parte, el carácter exhortativo de la petición se aminora con respecto a los enunciados anteriores en estos otros enunciados:

(6) Venga. A jugar. \#ResistenciaMovistar

(7) Venga, deje ese orgullo y seamos felices.

(8) Venga no le ruegue más, tenga dignidad.

(9) Venga, vamos a dejar claros unos conceptos a ver si así...

(10) Venga a rendir cuentas, por favor.

(11) venga usemos el HT \#tortugaton

En los enunciados anteriores las formas lingüísticas son, sobre todo, perífrasis verbales, subjuntivos con valor imperativo y la construcción a + infinitivo. En (10) la fórmula de cortesía negativa final (a través del enfocador de la alteridad por favor) aminora el carácter intensivo de venga. También, el carácter intensivo de venga se aminora en los enunciados irónicos, sobre todo los de tema político, los más frecuentes en Twitter:

(12) Venga, el que votó a Vox que lo diga, que le prometemos que no nos vamos a reír.

(13) Venga, pedid perdón a Rato y Fernández Díaz y disolveos. 
Es evidente la crítica social que encierran todos estos enunciados exhortativos-irónicos. Además de la función apelativa (con una finalidad comunicativa muy diferente a la de los ejemplos anteriores), es la función lúdica la que se hace presente. Los enunciados irónicos se hallan también fácilmente fuera del ámbito político:

(14) Venga le digo, ámeme

(15) Venga esos chistes de que Australia no está en Europa, que no cesen

(16) Venga no se tiren insultos. Solo es un juego, peleen cuando los manden a lavar loza, por fútbol qué, sean felices y buenos hinchas, OJO.

Otras veces la petición va implícita en lo dicho (los 200 RT) en una oración subordinada condicional, que funciona a modo de argumento (Venga + argumento + conclusión):

(17) Venga, si este tweet llega a 200 RT subo una imagen para que os hagáis una idea del RapPlay de Bloodborne. =)

E, incluso, el marcador sirve para introducir un enunciado de carácter publicitario no comercial:

(18) Venga, este año no esperes a julio para renovar el DNI y poder irte de vacaciones ¡Pide cita YA! Cómodo y rápido http://www.citapreviadni.es o 060

\subsection{Venga para transmitir ánimo}

Por otra parte, con venga se intenta transmitir ánimo en el destinatario. En este caso, el elemento lingüístico sumamente repetitivo en Twitter, relacionado directamente con la función apelativa, es el vocativo. El enunciado más neutro se conforma solo por el marcador y el vocativo (Venga + vocativo):

(19) ¡Venga América!

(20) iVenga Calero!

(21) Venga Tigueres!

Como Tanghe explica, el vocativo en combinación con los marcadores derivados de verbos de movimiento "sirve principalmente para atenuar un acto que podría amenazar la imagen social del oyente" (2016: 232). Para Edeso (2005), el vocativo se usa para indicar cortesía, autorreflexión por parte del hablante y para implicar al oyente. En los enunciados de este trabajo, en los que venga transmite ánimo o formula una petición, se trata de un vocativo implicativo y portador de la cortesía positiva. 
No obstante, lo más frecuente es que se hagan presentes en el mismo enunciado la expresión imperativa y el vocativo:

(22) Venga mi Julie!!! A darle con todo al Free Style, nuevos retos en @BailaSiPuedes \#teamVince 7 pm, Azteca 13

(23) Venga Barça, a marcar un gol más!!

(24) Venga Tigres, a poner en alto el nombre del fútbol mexicano, suerte

(25) iVenga, Pisarello, intenta quitarle la bandera a Mireia!

(26) Venga Vamily, tenemos que superar esta segunda fase de la competición! \#FandomOlympics \#TEAMVAMPS

(27) iVenga Rojiblancos! Sin parar

(28) Venga Águilas a remontar como campeones

Nótese cómo en los anteriores enunciados es muy frecuente la construcción a + infinitivo como expresión imperativa. En cuanto a la argumentación discursiva, al vocativo sigue la conclusión (Venga + vocativo + conclusión). No obstante, en otros enunciados lo frecuente es que al vocativo siga el argumento del acto exhortativo (Venga + vocativo + argumento), estando la conclusión implícita:

(29) Venga niños!!! Que ya es viernes !!!!

(30) Venga prima! Que todos estamos contigo!

(31) iQuillo, venga pa arriba que se te hace tarde...!

(32) Venga Víctor, este va a ser el negocio de tu vida, y seguro que lo haces bien \#Acacias

La ironía se sigue haciendo presente en la estructura lingüística de estos tuits. Como había ocurrido antes, el carácter intensivo de venga se aminora en los enunciados irónicos y de crítica social y política:

(33) Venga mama chilla un poco mas cuando hables por telefono k no me has despertado aun $x d d d$

(34) Venga rita ahora a tomar caloret por un tubo... Pero pagándotelo tu eh..

(35) Venga hombre, colaborad que estos intelectuales tienen que comer de algo.

En otros enunciados, se elude el vocativo y a venga le sigue inmediatamente el argumento (Venga + argumento): 
(36) Venga que a este ritmo tenéis animación cada finde.

(37) iVENGA QUE YA ES JUEVES MADRID ARDE! \#Feliz)ueves

(38) Venga! sólo quedan 4 días de campaña! \#SomosManuela

(39) Venga,nos espera un gran día!!

Por último, hay que hacer notar que la reduplicación del marcador es poco frecuente en Twitter, con o sin argumento:

(40) venga venga! El 2do tiempo es nuestro@Chivas!

(41) ¡VENGA,VENGA,VENGA!

\subsection{Venga para expresar desacuerdo}

Blas Arroyo (2001 [1998]: 9), al referirse al discurso oral, sostiene que "a ciertos actos de habla directivos como invitaciones, ofertas, propuestas, etcétera, le siguen otros actos reactivos cuyo objeto suele ser bien la aceptación, bien el rechazo". Aquí se trata del rechazo. Un rechazo que quiere influir en el comportamiento del destinatario, sin la expresión del argumento:

(42) VENGA CARAJO

Otras veces, a través del acto directivo de pregunta:

(43) Venga ¿Usted por qué no se traga su orgullo y viene y me habla?

(44) Venga, coño, ¿̇ese pelo es de verdad?

O a través de la expresión venga ya:

(45) Venga ya, que todo sabemos cómo va a acabar el \#diaDelOrgulloFriki

(46) Venga ya, que salga labrador \#TronoChicos

Y en muchas ocasiones lo que se pone de manifiesto es la ironía y mucha crítica social de tinte político:

(47) Venga, esas fotos de monjas votando no se van a hacer solas.

(48) Venga, Espe, que lo estás deseando: ¡LEVANTAMIENTO MILITAR!

(49) Venga "caloret", gracias por robar, incumplir, malgastar e insultar! Hasta nunca! ;) 


\section{El marcador discursivo conversacional venga en posición final del enun- ciado}

\subsection{Venga para transmitir ánimo}

En posición final, venga transmite sobre todo ánimo. Aquí caben tres posibilidades:

a) Lo más frecuente es que el marcador vaya seguido de un vocativo (Venga + vocativo):

(50) 67' Cambio: salió Omar Bravo, entró @DeNigrisg. iVenga Aldo! \#SiempreJuntos

(51) Muchas gracias por todo!!! Estoy muy orgulloso de mis jugadores y muy agradecido con la afición por apoyarnos cada partido! Venga mi tibu!!!

(52) Soberbia primera parte del Guadalajara. No hay que confiarse, está casi en la bolsa. venga@Chivas!!!

(53) las noches de Domingo son las más largas de toda la semana, ¡Venga Colegas!

(54) iQue locura en el Universitario! Tres minutos más y a semifinales ¡Venga @TigresOficial!

(55) Este momento quedará guardado en la historia del madridismo!! Rt si nunca lo olvidaras, venga madridistas!!

b) Que al vocativo siga una expresión imperativa (Venga + vocativo + expresión imperativa):

(56) 62' Cambio: salió @ @raullg8 entró @werofierro. iVenga Carlos, a darlo todo! \#SiempreLuntos

(57) —Cerati, ¿y si te pones algo de música? Ligera, de preferencia. —Ché, Enrique, la chispa adecuada, ¿te parece? —Venga, tío. -Dale.

c) O que el marcador ocupe por sí solo la posición final. En este caso, caben hacerse dos consideraciones importantes: que venga tenga suficiente fuerza ilocutiva (en estructuras exclamativas) o que dicha fuerza sea menos perceptible. De la primera consideración, valgan los tuits siguientes:

(58) Buen lunes, muy buena semana para todos, ya es hora, andando con actitud y de la buena, esa que motiva de inmediato, venga!!!.

(59) ¡Sábado de FINALES DE COPA! iiiVENGA!!!

(6o) te imaginas que este gh lo conduzca Solita? ADELANTE MIS VALIENTES, CAGUENSE A PALOS, VENGA!!!

Y, en cuanto a los enunciados en los que la fuerza ilocutiva es menos perceptible o que el emisor no ha tenido la intencionalidad de hacerla más palpable, he aquí estos ejemplos: 
(61) Buen sábado, buena mañana para todos, ya es un gran día, así que andando, arriba y con toda la actitud!!!!. Venga...

(62) No hay pex, @Chivas, metiendo un gol cada 5 minutos la hacen... Venga, sí se puede, venga...

(63) ¡Buen día!, buena semana, no importa como te sientas, tu arréglate, perfúmate y sal a conquistar la vida, venga.

En todos estos últimos enunciados venga marca la pre-conclusión de la conversación. Y se justifican, por tanto, una vez más, los grados de fuerza ilocutiva que, dependiendo de la situación comunicativa y el contexto lingüístico, manifiestan los enunciados en los que este marcador establece sus inferencias.

\subsection{Venga para introducir una petición}

La petición es poco frecuente cuando el marcador ocupa la posición final. Estos son algunos ejemplos de los escasos del corpus:

(64) Ya estas yendo pa er mercadona, uy pa el mercadona... Pa er corte inglés pa el mediamá pa el carrefú pa donde sea a comparar er disco venga

(65) Stannis... Hazme una pira de piedra... venga vamos a quemaaaaar algunos juegos free-toplay están hechos para sacar dinero a los jugadores ¡Venga, usad el hashtag!

\subsection{Venga para expresar desacuerdo}

Al igual que en posición inicial, lo que se pretende con esta función de venga es que el destinatario perciba la oposición, desacuerdo o rechazo del emisor. Hay que señalar, no obstante, que la función de venga como enfocador de la alteridad es muy poco frecuente en posición final. Estos son algunos ejemplos:

(66) DELINCUENTES, Mentirosos, Sinvergüenzas. Y la culpa es de Twitter? Venga Coño!!

(67) Bueno ya acortamos distancia con ayuda de Gallese. Abajo 2-1. Venga América carajo!

\section{El marcador discursivo conversacional venga en posición medial del enunciado}

\subsection{Venga para transmitir ánimo}

En posición medial es frecuente que el marcador se acompañe del vocativo: 
(68) Ya falta menos para ver a @JorgeBlancoG venga compadre ya estamos toda la familia listos!

(69) ¡Ahí está el ejemplo! Venga chavos, creo en ustedes... imás medallas para \#MEX

Otras veces, al marcador le sigue inmediatamente el argumento:

(70) Llegamos al sábado \#Guerreros! Venga, que el fin de semana apenas comienza \#GuerrerosMásQueNunca

\subsection{Venga para introducir una petición}

Cuando venga introduce una petición en posición medial, resulta muy difícil delimitar la petición del ánimo, como puede comprobarse en los siguientes tuits, en los que la petición y el ánimo se amalgaman:

(71) 400 favs a este tuit y hago un \#HoróscopoDeYisus venga shavales ya sabéis favs y rt a tope.

(72) y en cuanto voy pal rincón, venga quillo, vamonós, ya sabes quién ha llegao y hay que andarse con cuidao.

(73) Faltan chistes de su regreso a clases, venga, están a tiempo.

(74) Volvemos a estar en el primer puesto!!venga seguid votando por los chicos en \#MTVHottest One Direction-Z

\subsection{Venga para expresar desacuerdo}

Por otra parte, no faltan los tuits — como se ha visto en enunciados anteriores - donde se hace presente la crítica social y política. En estos casos, venga funciona como enfocador de la alteridad, expresando el desacuerdo. Los enunciados son frecuentes:

(75) UPyD abandona todos los procesos judiciales en los que estaba. Venga, que solucionen lo de las preferentes de Bankia Podemos y Ciudadanos

(76) Oye Marhuenda lo de decir algo en TV y no cumplirlo está muy feo. Venga! Danos una alegria! \#NuevaPolíticaARV

(77) - Tigres!! Leones!! TODOS SOMOS CAMPEO00000NES!! - Venga señora Aguirre. Baje del atril.

(78) ¿Pero que coño? Venga... id procesando que ESTO ES NORMAL, lo anormal es lo vuestro. \#GaysNoMerecenMedallas 


\section{Fórmulas conversacionales con venga}

Se estudian, a continuación, algunas fórmulas conversacionales con venga portadoras de la función apelativa.

\subsection{Venga, vamos / Vamos, venga}

Se estudia, en primer lugar, la combinatoria de venga con vamos. Para Tanghe (2008-2009: 99), "parece que vamos refuerza el valor exhortativo de venga y viceversa". Téngase en cuenta que el marcador vamos deriva del uso subjuntivo de esta forma en el español medieval y clásico, según el análisis exhaustivo de González Ollé (2002). Cortés y Camacho (2005: 177) incluyen tanto jvenga! como ivamos! entre "los marcadores cuya intención es motivar o cambiar la actitud del interlocutor". Como Chodorowska-Pilch (1999: 344-345) señala, vamos facilita la modulación de la proximidad interpersonal expresando metafóricamente buena disposición y cortesía hacia el interlocutor. En cuanto a la fórmula conversacional que nos ocupa, vamos funciona como intensificador de venga. Lo normal es que venga siga a vamos, coocurrencia que puede ocupar cualquier posición dentro del enunciado, intensificando el ánimo:

(79) VAMOS VENGA!!!

(80) Vamos vamos venga venga, lo estais deseando!!

(81) Sacame a tu Charizard, VAMOS, VENGA, HAZLO, I DARE YOU, I DOUBLE DARE YOU. Que Twitter censure tíos con abdominales...vamos venga, no es contenido sensible.

La petición:

(82) I want nudes. Vamos, venga.

O el desacuerdo:

(83) En mi vida he hablado con Dalas y aquí la gente ya se saca que le he enviado fotos. Claro que si señores. Vamos. Venga.

Es menos frecuente que vamos siga a venga, ocupando esta construcción cualquier posición en el enunciado, intensificando siempre el ánimo:

(84) Venga vamooos!!

(85) Venga vamos con todo!!! Misael Rodríguez hasta el momento el único medallista olímpico mexicano es Tuzo!!! 
(86) Deberías probar el vino que tengo aunque sea.. Venga vamos, no se hable más

(87) Yo: *twitteo de día* Los que sigo: A TUITEAR DE NOCHE VENGA VAMOS

\subsection{Venga, va}

Por otra parte, es frecuente la fórmula conversacional Venga, va. Según Hernández, este va en español es un caso de interferencia lingüística del catalán, cuyo posible origen "bien puede ser producto de la confusión, muy común en el español de Cataluña, entre los verbos ir y venir del español y los verbos anar y venir del catalán” (1997: 7). Por otra parte, según esta autora, dicha interferencia "se podría explicar de una manera mucho más simple y es que la productividad del va catalán en determinados contextos ha llegado a contaminar e invadir un terreno que en español en principio era del venga (y que también pueden ocupar el vamos, el anda y otras expresiones similares)" (1997: 8). En estos enunciados con va (frente a los anteriores con vamos) predomina la petición. Al igual que en el apartado 2.1., el acto de habla directivo exhortativo se consigue por la fuerza que transmiten las formas verbales imperativas:

(88) Venga va hacedme preguntas!: ) yo os contesto todas

(89) Venga va enviad las mayores frikadas \#DiaDelOrgulloFriki

O a través del subjuntivo con valor imperativo:

(90) Venga va que lo escruten todo que me quiero ir a dormir!!!!

O por medio de la hipótesis implícita en una oración subordinada condicional:

(91) Venga va, si este tweet llega a 50 FAV cuelgo el pick que llevamos en el grupo privado para las 05h.

(92) Venga va, si llegamos en este Tweet a unos 300 \#FAVS, sorteo esta cuenta con estos jugadores:)

Por otra parte, esta fórmula conversacional, más raramente, puede intensificar desacuerdo, como en el siguiente tuit en el que se acompaña de un vocativo:

(93) Venga va @realmadrid, la broma de Casillas y Benitez ha sido graciosa pero dejad de hacer el tonto ya 


\subsection{Venga, anda / Anda, venga}

Para Moreno García, venga a veces alterna con anda, como imperativos lexicalizados, "para animar a la acción" y también "para mostrar incredulidad o negar lo afirmado por el otro" (2007: 18). En Twitter se trata, sobre todo, de un enfocador de la alteridad y expresa desacuerdo. Las dos coocurrencias se documentan por igual en Twitter. Venga, anda ocupa cualquier posición en el enunciado:

(94) Venga anda putilla vete ya a la universidad

(95) Venga anda, vete a un asilo, donde se tragan las tonterías que dices, que por aquí no cuela, en serio;)

(96) Pero @isco_alarcon si, venga anda dile a Tito Floren que te haga la maleta y te vengas para Málaga!

(97) Que salvame la largue ahora me parece ridículo... No sabía a quien llevaba??... Venga anda

Anda, venga, no obstante, suele ocupar la posición inicial:

(98) Anda venga ya cabrón, que yo era un fan de estos de pega que se tatúa las reliquias y poco más.

(99) Anda, venga vamos, José Luí; más vino y menos cava, que se te ven las costuricas, cariño!!

(100) Datos objetivos | ¿Le viene bien sólo a Aramís? ¡ANDA VENGA! Hipocresía barata.

En esta posición inicial anda, venga puede también intensificar la petición:

(101) anda venga va por favor anda venga va por fa.

(102) anda venga preguntarme cosasss

(103) Anda venga, si me compráis el iPhone os regalo a mi perro también que para nada se porta mal.

Nótese cómo en (101) confluyen la petición y el ánimo.

\subsection{Pues venga}

En la fórmula conversacional pues venga, pues es un consecutivo de clara referencia anafórica, que intensifica el valor de venga incitando al destinatario a la acción. Pues intensifica sobre todo la petición, potenciando la cortesía positiva, o mitigando la descortesía. Ocupa en el discurso, mayoritariamente, la posición medial: 
(104) Es TT \#ColaboraConPodemos, jajaja, pues venga, a darle!!!

(105) Por cierto, ¿sabéis que tenéis que mimar mucho no? Pues venga, móviles apagados y a besar con locura. ¡Hasta mañana!

(106) Tenéis plan para esta noche? ...pues venga, a la sala Totem i

(107) ¿Es que no nos gusta verlos así? Pues, venga, conéctate a http://losreyesmagos.tv ya.

(108) pues venga ivan, dale buenas noches a tu gaviotita de peluche y a dormir. Y recuerda sacarte las bolitas chinas

También, en menor medida, puede intensificar el ánimo:

(109) Pues venga, con dos huevos al juzgado de guardia.

\section{Conclusiones}

En cuanto a las conclusiones, se detallarán, en primer lugar, los resultados de esta investigación, para pasar, a continuación, a señalar las líneas de investigación que a partir de aquí se abren. En esta investigación se han conseguido los siguientes resultados:

a) El marcador conversacional venga, ampliamente usado en la red social Twitter, desempeña el valor más próximo al valor conativo de la forma imperativa originaria, ocupando mayoritariamente la posición inicial del enunciado. De su uso imperativo originario derivan los valores de ánimo, petición o desacuerdo. Los relacionados directamente con la función apelativa son los dos primeros. No obstante, aunque en el desacuerdo predomine la función expresiva, también se intenta influir en el comportamiento del destinatario. Por otra parte, no resulta fácil, a veces, delimitar la petición del ánimo, al darse conjuntamente en el mismo enunciado.

b) En posición inicial, se usa en intervenciones no reactivas, para transmitir ánimo o introducir una petición; y en intervenciones reactivas, para expresar desacuerdo. En posición final se usa, sobre todo, para transmitir ánimo. Y en posición medial transmite ánimo, introduce una petición o expresa desacuerdo. De estas tres funciones, por tanto, ocupa un primer lugar la expresión del ánimo, siendo estos enunciados buenos ejemplos de cortesía positiva.

c) La fuerza pragmática del acto ilocutivo depende de los intensificadores que acompañen al marcador: el vocativo implicativo — sobre todo para transmitir ánimo-y las expresiones imperativas son los elementos lingüísticos intensificadores de la función apelativa. sin embargo, el carácter intensivo de venga se aminora en los enunciados irónicos, mayoritariamente de tema político. En estos enunciados, además de la función apelativa, se 
hace presente la función lúdica. En cuanto a la argumentación discursiva, predominan los enunciados en los que a venga sigue la conclusión, sin la expresión del argumento.

d) Por último, otros marcadores discursivos en coocurrencia con venga sirven para intensificar, sobre todo, el ánimo (Venga, vamos y Vamos, venga); para intensificar, sobre todo, una petición (Venga, va y Pues venga); y para intensificar el desacuerdo y, en menor medida, para intensificar una petición (Venga, anda y Anda, venga).

Por otra parte, a partir de este estudio se abren otras líneas de investigación relacionadas con el uso de otros marcadores conversacionales en la red social Twitter, resultado del proceso de gramaticalización que han experimentado algunos verbos de movimiento españoles como, por ejemplo, vamos, vaya o anda, y cuyos resultados habría que contrastar con los obtenidos en esta investigación.

\section{Bibliografía citada}

Alonso-Cortés, Ángel, 1999: "Las construcciones exclamativas. La interjección y las expresiones vocativas" en Ignacio Bosque y Violeta Demonte (dirs.): Gramática descriptiva de la lengua española, Madrid: Espasa, 3993-4050.

BARAN, Marek, 2010: Emotividad y convención sociopragmática. Una contribución al estudio del ethos comunicativo de la comunidad hispanohablante peninsular, Łódź: Wydawnictwo Uniwersytetu Łódzkiego.

BARAN, Marek, 2014: “La subjetivización y las dinámicas interaccionales”, Studia Romanica Posnaniensia XLI/1, 3-16.

Blas Arroyo, José Luis, 2001 [1998]: "Un caso de variación pragmática sobre la ampliación significativa de un marcador discursivo en el español actual. Aspectos estructurales y sociolingüísticos" [http://www.anmal.uma.es/Numerog/Arroyo.htm, fecha de consulta: 4 de octubre de 2016].

Briz, Antonio, Salvador Pons Bordería y José Portolés (coords.), 2008: Diccionario de partículas discursivas del español [http://www.dpde.es, fecha de consulta: 29 de septiembre de 2016].

Calsamiglia Blancafort, Helena, y Amparo Tusón Valls, 1999: Las cosas del decir. Manual de Análisis del discurso, Barcelona: Ariel.

Casado Velarde, Manuel, 1995: Introducción a la gramática del texto español, Madrid: Arco/ Libros.

Chodorwska-PILCH, Marianna, 1999: "On the polite use of vamos in Peninsular Spanish", Pragmatics 9 (3), 343-356. 
CiarRa Tejada, Alazne, 2016: Marcadores discursivos conversacionales: análisis de su uso en corpus orales y aplicación didáctica en español como lengua extranjera, Salamanca: Ediciones de la Universidad.

Cortés Rodriguez, Luis, 2001: "Conectores, marcadores y organizadores como elementos del discurso" en José Jesús de Bustos Tovar y otros (eds.): Lengua, discurso, texto. I Simposio Internacional de Análisis del Discurso I, Madrid: Visor, 539-550.

Cortés, Luis, y María Matilde Camacho, 2005: Unidades de segmentación y marcadores del discurso, Madrid: Arco/Libros.

Daniels, Kelsey, 2014: "On the grammaticalization of «venga» 'come' as a discourse marker in Peninsular Spanish" en Maud Devos y Jenneke van Der WaL (eds.): «Come» and «go» off the beaten grammaticalization path, Berlin/Boston: De Gruyter Mouton, 219-248.

Edeso Natalías, Verónica, 2005: "Usos discursivos del vocativo español”, Español Actual 84, $123-142$.

GonzÁlez Ollé, Fernando, 2002: "Vamos: un conector coloquial de gran complejidad" en M. a Antonia Martín Zorraquino y Estrella Montolí Durán (coords.): Los marcadores del discurso. Teoría y análisis, Madrid: Arco/Libros, 117-135.

Haverkate, Henk, 2003: "El análisis de la cortesía comunicativa: categorización pragmalingüística de la cultura española” en Diana Bravo (ed.): Actas del I Coloquio del programa EDICE. La perspectiva etnocentrista de la cortesía: identidad sociocultural de las comunidades hispanohablantes, Estocolmo: Universidad de Estocolmo, 60-70.

Hernández García, Carmen, 1997: “El va en español, un caso de interferencia del catalán”, Espéculo 6, 1-10 [https://pendientedemigracion.ucm.es/info/especulo/numero6/c_hernan.htm, fecha de consulta: 11 de octubre de 2016].

LuQue Toro, Luis, 2009: “Aspectos pragmáticos y cognitivos de los marcadores discursivos de las formas verbales ‘andar', 'ir' y ‘venir' ”, Léxico Español Actual II, Venezia: Cafoscarina, 131-144.

Matte Bon, Francisco, 1992: Gramática comunicativa del español II, Madrid: Edelsa.

Monjour, Alf, 2006: “'Pasad, pasad' - 'Kommen sie bitte rein'. Pedro Almodóvar, los actos de habla y la comparación intercultural” en Martina Schrader-Kniffi (ed.): La cortesía en el mundo hispánico, Madrid: Vervuert Iberoamericana, 15-42.

Moreno García, Concha, 2007: "Gramática y atención a la forma: sentido y sensibilidad (I)", Mosaico 19, 16-21.

TANGHE, Sanne, 2008-2009: Sobre algunas interjecciones derivadas de los verbos de movimiento: anda, vamos, vaya y venga [http://lib.ugent.be/fulltxt/RUG01/001/414/921/ RUG01001414921_2010_0001_AC.pdf, fecha de consulta: 14 de octubre de 2016]. 
TAnghe, Sanne, 2016: Marcadores derivados de verbos de movimiento. Una aproximación cognitiva a su polifuncionalidad, Beihefte zur Zeitscrift für Romanische Philology: Edition Niemeyer. 